\title{
Ovulatory changes in the wall at the apex of the human Graafian follicle
}

\author{
H. Okamura, A. Takenaka, Y. Yajima \\ and T. Nishimura
}

\begin{abstract}
Department of Obstetrics and Gynecology, Kyoto University School of Medicine, Sakyoku, Kyoto, Japan
\end{abstract}

\begin{abstract}
Summary. The apical wall was studied ultrastructurally in 16 human follicles at various stages of development. In the theca externa and tunica albuginea at the apex of growing follicles, fibroblasts with little cytoplasm and abundant collagen fibres were observed. In the mature follicles, the fibroblastic cytoplasm was well developed, rich in lysosome-like granules and contained peripheral multivesicular structures. Intercellular collagen fibres were sparse. It is suggested that the collagen fibres are digested by the content of lysosomal granules and multivesicular structures, thus aiding follicular rupture.
\end{abstract}

\section{Introduction}

Various aspects of the mechanism of follicular rupture at ovulation have been studied, e.g. the roles of follicular contractility, proteolytic enzymes, histamine and prostaglandins (Weir \& Rowlands, 1977). Degradation of collagenous connective tissue in the apical portion of the preovulatory follicular wall (Espey, 1967) and collagenolytic activities in the follicle (Espey, 1974) have been demonstrated in the rabbit. In the present study, we investigated the ultrastructure of collagen fibres and fibroblasts in the tunica albuginea, theca externa and theca interna at the apex of the human ovarian follicle.

\section{Materials and Methods}

The material was obtained from 56 women aged 30-40 years who were undergoing total hysterectomy for uterine myomata or carcinoma of the uterine cervix. The ovaries were carefully examined and the follicle which was growing in that menstrual cycle was dissected out from each ovary and fixed immediately for the ultrastructural study. The follicles used were as follows; 3 follicles with a diameter of 1-2 mm (early proliferative); 3 with a diameter of 5-6 mm (mid-proliferative); 3 with a diameter $>10 \mathrm{~mm}$ (preovulatory); 3 follicles harvested $29 \mathrm{~h}$ after the injection of 5000 i.u. hCG (Mochida Pharm. Co., Tokyo, Japan) preceded by treatment with clomiphene citrate (Clomid: Shionogi Pharm. Co., Osaka, Japan) $(50 \mathrm{mg} /$ day for 5 days starting on Day 5 of the cycle); 3 follicles which had just ruptured and still had broken tissue at the apical region; and 1 follicle harvested $22 \mathrm{~h}$ after the injection of 5000 i.u. hCG preceded by 150 i.u. hMG (Humegon: Nippon Organon K.K., Tokyo, Japan) for 7 days starting on Day 5 of the cycle. The purpose of the treatments was explained to the patients and their written consent was obtained before treatment and the project was approved by our ethical committee.

From each follicle, the apical region was excised into a strip and fixed in chilled $6.25 \%$ glutaraldehyde in $0.1 \mathrm{M}$-cacodylate buffer ( $\mathrm{pH} \mathrm{7.4)}$ containing $8 \%$ sucrose for a period of $2 \mathrm{~h}$. Postfixation was carried out in a chilled $1 \%$ solution of osmium tetroxide. The tissue blocks were 
stained in saturated uranyl acetate solution, dehydrated in a series of graded alcohols followed by propylene oxide, then embedded in Epon 812. From these plastic blocks, $1 \mu \mathrm{m}$ sections were cut, stained in toluidine blue and examined with the light microscope. Ultrathin sections were cut with a glass knife on a Porter-Blum IIB ultramicrotome, stained with lead citrate solution, and examined in an electron microscope (Hitachi HU-11D) at $75 \mathrm{kV}$.

\section{Results}

The apical portion of the follicle wall is composed of 6 layers, namely, from the outside inward, germinal epithelium, tunica albuginea, theca externa, theca interna, basement membrane and a granulosa layer. Since the theca interna and granulosa layers mainly consist of epithelial cells with little stromal tissue, and abundant collagen bundles are observed in the tunica albuginea and theca externa, the present observations were restricted to only these two layers. In the follicles at the early follicular phase with 1-2 mm diameter, the tunica albuginea and theca externa had prominent collagen bundles. Fibroblasts with little cytoplasm were present. In the tunica albuginea and theca externa of the growing follicles at the mid-follicular phase $(5-6 \mathrm{~mm}$ diam.), fibroblasts could be seen among abundant collagen bundles but they still had poorly developed cytoplasm (Pl. 1, Fig. 1). Granulosa cells of the same follicles had round nuclei with prominent nucleoli, small mitochondria with lamellar cristae, moderately developed rough endoplasmic reticulum and free ribosomes (Pl. 1, Fig. 2) and confirmed that this follicle was actually growing. The granulosa cells of all the follicles were studied to confirm these characteristics and ensure that only growing, i.e. not atretic, follicles were used. In the theca externa of the apical portion of

\section{PLATE 1}

Fig. 1. A growing follicle (5-6 mm diam.) showing fibroblasts (F) with little cytoplasm in the theca externa and abundant collagen fibres $(\mathrm{Co}) . \times 5600$.

Fig. 2. Granulosa cells in the same follicle as that shown in Fig. 1 have round nuclei $(N)$ with prominent nucleoli (No), small mitochondria (m) with lamellar cristae, rough endoplasmic reticulum (rER) and dense granules $(\mathrm{g})$. The basement membrane is shown by arrows. $\times 2700$.

Fig. 3. In the theca externa of the apical portion of a mature follicle ( $12 \mathrm{~mm}$ diam.), collagen bundles are infrequent, but fibroblasts $(F)$ have well developed cytoplasm with numerous mitochondria, rER and granules of low electron density $(\mathrm{g}) \times 3200$.

Fig. 4. In a preovulatory follicle from a woman treated with clomiphene and hCG, a fibroblast in the theca externa has a well developed Golgi apparatus (G) and rER. Vesicular structures (V) appear in the peripheral cytoplasm around which the stromal ground substance has become electron-lucent. $\mathbf{N}=$ nucleus. $\times 6400$.

\section{PLATE 2}

Fig. 5. In the theca externa of a preovulatory follicle after treatment with hMg and hCG, collagen bundles are scarcely observed, but the fibroblasts have a well developed cytoplasm filled with numerous mitochondria (m), rER, lysosome-like granules (Ly) and granules of low electron density $(\mathrm{g}) \times 2600$.

Fig. 6. The germinal epithelium (GE) and tunica albuginea (TA) of a preovulatory follicle after treatment with clomiphene and hCG are shown. Collagen bundles $(\mathrm{Co})$ are sparse and fibroblasts have abundant cytoplasm. Early signs of degeneration are seen in the germinal epithelium. $\times 2100$.

Fig. 7. The germinal epithelium (GE) and tunica albuginea (TA) of the follicle wall shortly after rupture. In the tunica albuginea, scattered and disintegrated fibrillar substances (f) are observed. No collagen fibres could be seen. $\times 8000$.

Fig. 8. Multivesicular structures (MV) are observed in the periphery of fibroblasts in the theca externa of the follicle after rupture. $\times 5600$. 
PLATE 1
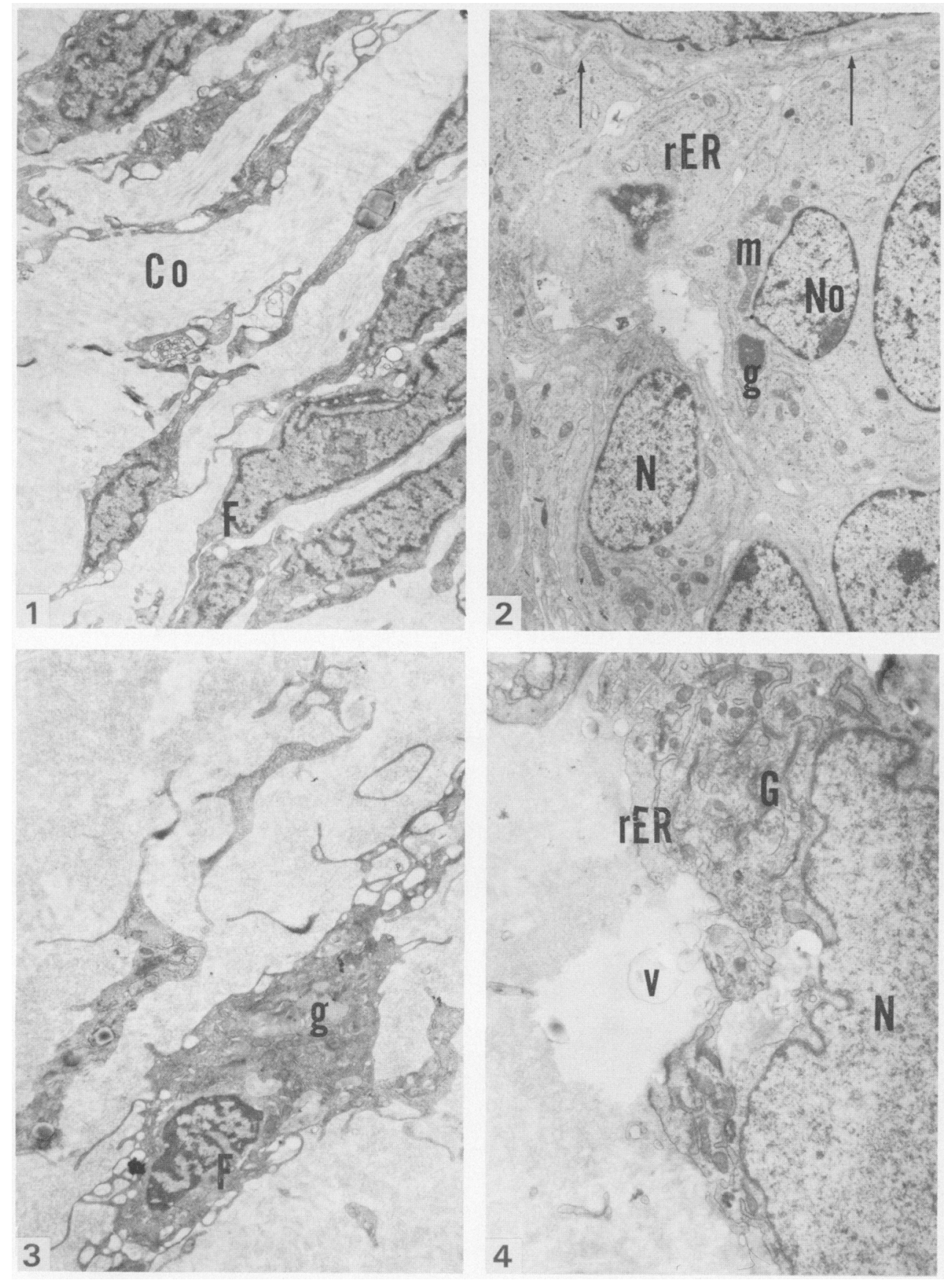

(Facing p. 154) 


\section{PLATE 2}

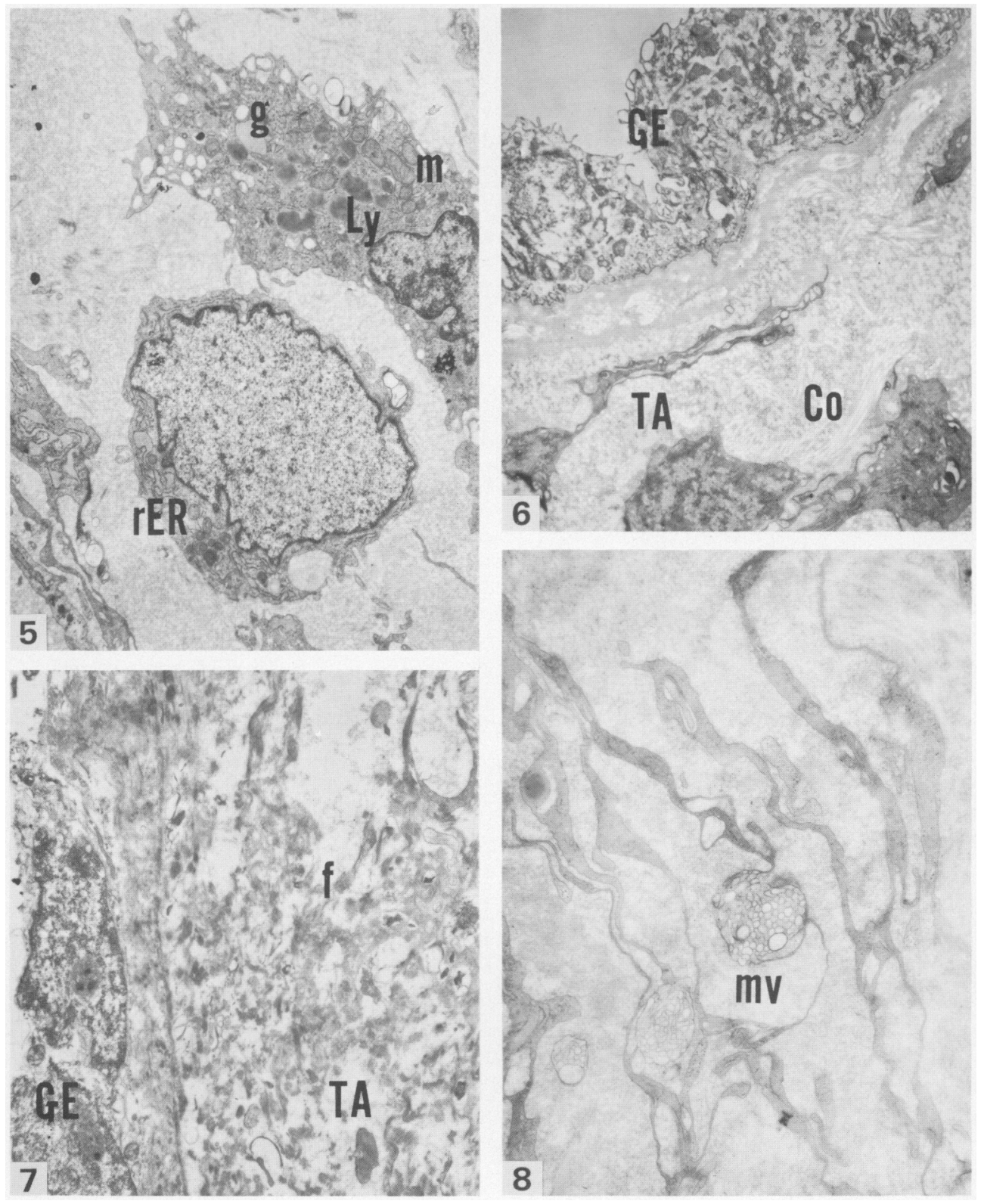


mature follicles (diam. $>10 \mathrm{~mm}$ ), collagen bundles were hardly ever observed. Fibroblasts in this layer had well developed cytoplasm with abundant rough endoplasmic reticulum and granules of low electron density (Pl. 1, Fig. 3). In the preovulatory follicles, fibroblasts in the theca externa had well developed Golgi apparatus, rough endoplasmic reticulum and lysosome-like granules. These fibroblasts were particularly prominent in the follicles from hCG- and hMG-treated women (Pl. 2, Fig. 5). In some fibroblasts, vesicular structures were observed in the peripheral cytoplasm around which the stromal ground substance had become electron-lucent (P1. 1, Fig. 4). In the tunica albuginea of the preovulatory follicles, collagen bundles were sparse and the fibroblasts present had well developed cytoplasm. In the germinal epithelium, cells with ballooned cytoplasm and nuclei (Pl. 2, Fig. 6) were seen, indicating early cellular degeneration. After the rupture, the germinal epithelial cells had lost much of their cytoplasm, and the tunica albuginea was occupied by scattered fibrillar substance without any collagen fibres (Pl. 2, Fig. 7). In the theca externa, numerous multivesicular structures were observed round the edges of the fibroblasts, the fibrillar substance was abundant and collagen bundles were infrequent (Pl. 2 , Fig. 8).

\section{Discussion}

The observation that prominent collagen bundles in the layers of tunica albuginea and theca externa in growing follicles have almost disappeared before ovulation is in agreement with findings for the rabbit by Fspey (1967). The disappearance of the collagen bundles and the existence of a fibrillar substance in recently ruptured follicles indicate that the collagen bundles may have been enzymically degraded into fibrils. The ultrastructural characteristics of the fibrobiasts in the theca externa of preovulatory follicles indicate that active protein synthesis is occurring, probably with enzymes contained in the lysosome-like granules. Since collagenolytic cathepsins are lysosomal enzymes, these ultrastructural features suggest the increased activity of collagenolytic enzymes in preovulatory follicles. This deduction from present morphological observations is in agreement with our studies of cathepsin $B_{1}$ activity in the human preovulatory follicles (Yajima, Motohashi, Takenaka, Okamura \& Nichimura, 1980). The multivesicular structures observed in the peripheral cytoplasm of fibroblasts in human follicles before and after ovulation are similar to those reported for rabbit preovulatory follicles (Espey, 1967) and human binovular follicles (Papadaki, 1978). They appear concomitantly with collagen degradation and could be carriers of the collagenolytic enzymes to the peripheral cytoplasm.

We conclude that follicle rupture at ovulation in women involves enzymic processes, particularly degradation of collagen, the stages of which have been demonstrated in the present study.

\section{References}

Espey, L.L. (1967) Ultrastructure of the apex of the rabbit graafian follicle during the ovulatory process. Endocrinology 81, 267-276.

Espey, L.L. (1974) Ovarian proteolytic enzymes and ovulation. Biol. Reprod. 10, 216-235.

Papadaki, L. (1978) Binovular follicles in the adult human ovary. Fert. Steril. 29, 342-350.
Weir, B.J. \& Rowlands, 1.W. (1977) Ovulation and atresia. In The Ovary, 2nd edn, vol. 1, pp. 265-301. Eds S. Zuckerman \& B. J. Weir. Academic Press, New York.

Yajima, Y., Motohashi, T., Takenaka, A., Okamura, H. \& Nishimura, T. (1980) Activities of collagenolytic enzymes in the human ovary. Acta obstet. gynec. jap. 32 (in press).

Received 23 April 1979 